MATEC Web of Conferences 31, 15004 (2015)

DOI: $10.1051 /$ matec conf/ 20153115004

(C) Owned by the authors, published by EDP Sciences, 2015

\title{
An Improved GLRT Method for Target Detection in SAR Imagery
}

\author{
Yingyun Ju,a ${ }^{1, \text { }}$ Peng Fu, Xin Zhou, Rongkun Peng \\ ${ }^{1}$ College of Automation Engineering, Nanjing University of Aeronautics and Astronautics, Nanjing 211106, Jiangsu, China
}

\begin{abstract}
Automatic ground vehicle detection based on SAR imagery is one of the important military applications of SAR. A region-based generalized likelihood ratio test (GLRT) method is proposed in this paper, and this method combines the GLRT detection theory and image segmentation technology. First, the SAR imagery is roughly segmented as land clutter region and potential target region through the split and merge procedure often used for processing the original images. Then, based on the segmentation results, the reasonable statistical models for the data in the two regions are built respectively. Finally, with the knowledge of statistical characteristics of clutter and target, GLRT detection method is applied to the each pixel in the potential target region to obtain more accurate detection results. Experimental results based on real SAR data show that the proposed method can effectively detect the ground vehicle targets from the land clutter with excellent accuracy and speed.
\end{abstract}

Keywords: SAR Imagery, Target Detection, Image Segmentation, Statistical Model, GLRT

\section{Introduction}

Synthetic Aperture Radar (SAR) is a kind of active imaging sensors, with its all-weather, all-time capabilities, etc, has been widely used in civil and military areas[1]. As a preliminary basis of target detection, target detection plays a vital role in subsequent target identification, recognition and tracking steps, so its research and development has been received widespread attention.

Because of the influence of factors such as the complexity of the ground scene, environmental change, coherent spot noise and so on, SAR images are very nonuniform, which results in a large number of false alarm. At present, the Constant False Alarm Rate (CFAR) method derived from Bayesian decision theory is one of the most widely used detection methods in SAR image target detection areas. Since Novak [2] summarized the good performance of Lincoln laboratory's two-parameter CFAR detection algorithm, the follow-up developments of CFAR detection algorithm appeared in large numbers to raise the CFAR performance such as accuracy and speed. Among them, CA-CFAR, GO-CFAR, SO-CFAR and OS-CFAR are four basic types of CFAR detectors. The later detectors are mostly a combination or variants of these four types. The famous VI-CFAR (Vector IndexCFAR) detector is a combination of these four detectors, which can keep good detection performance in various kinds of clutter backgrounds [3].

However, CFAR detection algorithms select the threshold without considering the target statistical properties, so it is actually a sub-optimal Bayesian decision method [4]. The other non-CFAR detection methods include generalized likelihood ratio method (GLRT) [5], based on neural networks [6], and detection algorithm based on support vector machines [7]. Collectively, these non-CFAR algorithms generally perform better, but the need of priori information and slow calculation speed restrict the promotion of these algorithms. Among them, GLRT detection uses global threshold, and has less computational cost. However, due to that GLRT detection algorithm needs to know the statistical distribution of the target and background, which is generally difficult to know in practice, the GLRT algorithm is greatly limited.

In this paper, a GLRT region-based target detection method is proposed for the problem of military vehicle detection in complex scenes. The method combines image segmentation technique and GLRT target detection algorithm. It first segments SAR image to clutter region and potential target region quickly and roughly by applying commonly used image segmentation and clustering methods. Then, appropriate statistical models for these two regions are established respectively. Through the above two steps, the problem that the target statistical properties of SAR image are difficult to obtain is solved. On the basis of the already known background and target statistical properties, a more accurate GLRT detection algorithm is used to further determine the target in potential target area. The actual data is processed in experimental part. And then this method is compared with other classical VI-CFAR detection methods. The results show that the proposed method can detect military

\footnotetext{
a Corresponding author: juyingyun@126.com
} 
targets from terrestrial scenes effectively, accurately and quickly.

\section{Data modeling of target region and background region}

Metal vehicle targets have strong radar echoes which show as larger amplitude value in SAR image compared with the background. Based on this phenomenon, a simple threshold technique is used to select all the possible metal target pixels. Then a clustering process is used to filter out the obvious target. After the process mentioned above, the cluster region and potential target region can be roughly separated. Then by analyzing the statistical properties of the two regions, suitable statistical models are established respectively.

Moment-preserving law is an automatic multithreshold selection algorithm commonly used in image segmentation. The law based on invariant properties of moments can automatically determine multi-threshold with low time-consuming. For a SAR image which only contains these two regions, the $i$-th moment is defined as:

$$
m_{i}=\frac{1}{n} \sum_{x} \sum_{y} f^{i}(x, y) \quad i=1,2,3 \cdots
$$

where $n$ denotes the total number of pixels, $f(x, y)$ is the amplitude value at $(x, y)$. Defining $m_{0}=1$ and supposing that the final best amplitude threshold is $T h^{*}$, we can achieve the SAR binary image segmentation task. By using $z_{0}$ and $z_{1}$ to represent the binary amplitude value after the segmentation, the first three moments can be defined as:

$$
m_{i}^{\prime}=\sum_{j=0}^{1} p_{j}\left(z_{j}\right)^{i} \quad i=1,2,3
$$

where $p_{0}$ and $p_{1}$ denote the proportion of $z_{0}$ and $z_{1}$ in the whole binary image. On the other word, they indicate the ratio of higher or lower pixels than the threshold $T h^{*}$ in the original image. Then we can get that

$$
p_{0}+p_{1}=1
$$

According to moment-preserving law, the result should remain each moment equally before and after the segmentation, which means that:

$$
\begin{gathered}
p_{0} z_{0}^{0}+p_{1} z_{1}^{0}=m_{0} \\
\vdots \\
p_{0} z_{0}^{3}+p_{1} z_{1}^{3}=m_{3}
\end{gathered}
$$

By solving equations (4), $p_{0}$ is obtained Then the best threshold $T h^{*}$ can be defined by the equation shown as:

$$
p_{0}=\frac{1}{n} \sum_{f_{j} \leq T h^{*}} n_{j}
$$

where $n_{i}$ denotes the number of pixels with an amplitude value $f_{i}$. According to the threshold $T h^{*}$, we can obtain two regions named $\mathrm{A}$ and $\mathrm{B}$ which contain pixels with the higher or lower amplitude value than $T h^{*}$ respectively.
Then region A can be determined as the potential target region.

Because of SAR image containing serious noise, many isolated pixels or small regions present in the segmentation result by using moment-preserving law. These pixels or regions are obviously false target. So it is necessary to use clustering process to filter them for a more concentrated and smooth result. An N-nearest neighbor method is used to deal with this problem. The basic thought of this method is: for a sample whose class to be judged, if most of the $\mathrm{N}$ adjacent samples in the feature belongs to a class, then the sample belongs to this class. As shown in Figure 1, supposing that $x$ denotes an isolated pixel or region in the A region, if there are more than $n$ surrounding pixels belong to region $\mathrm{A}$, then $x$ belongs to region A. Otherwise it belongs to region $\mathrm{B}$. In this paper, $N=8$ and $n=4$.

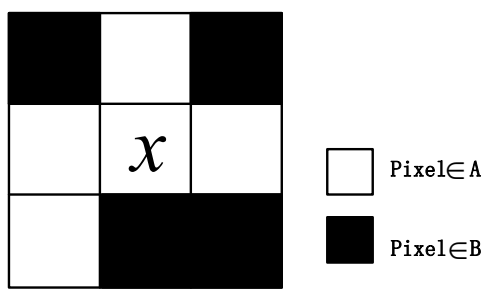

Fig.1 Schematic diagram of 8-neighbors

By the processes mentioned above, we can roughly depart the image to the potential target region $\mathrm{A}$ and cluster region B. The research presented in [8] shows that in the most SAR statistical models, $\mathrm{K}$ distribution is the most approaching distribution of land cluster and Lognormal distribution is the most approaching distribution of metal targets. Thus $\mathrm{K}$ and Lognormal distribution are used to model the land cluster and metal targets respectively. The specific mathematical expressions and the parameter estimation methods of them can be search in [9] and [10].

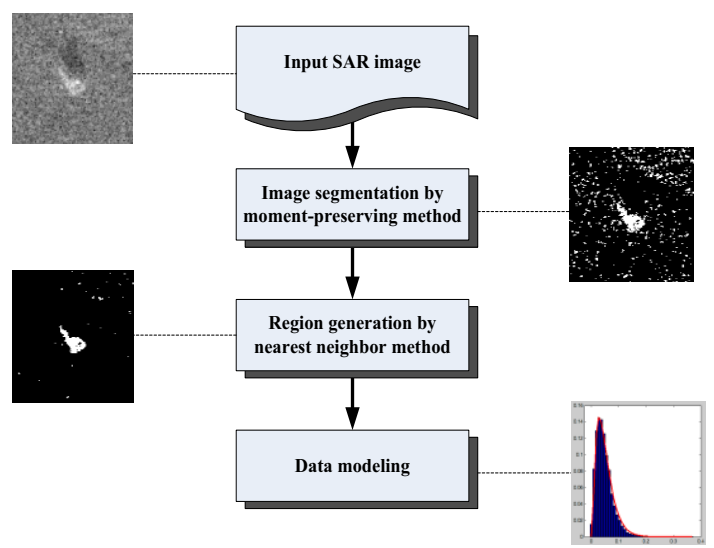

Fig.2 Flow chart of background \& target data modeling

Figure 2 is the flow chart of the modeling process of the target region and the cluster region in SAR image. The whole SAR image data modeling processes are shown as follows: 
1) Image segmentation. The optimal threshold is determined automatically to select all possible metal target area by using moment-preserving law.

2) Region generation. N-nearest neighbor clustering method is used to deal with the segmentation result in Step (1), filtering obviously false targets.

3) Data modeling. $K$ distribution model for land clutter region and Lognormal distribution model is established for potential target region.

\section{GLRT target detection}

In the above section, the SAR image is roughly segmented to a land clutter region and potential target region. Also the Lognormal model for the potential target region and $\mathrm{K}$ distribution model for the land clutter region are established. And the model parameters are estimated respectively. Under this condition, the generalized likelihood ratio (GLRT) detection method can be used to further identify whether the pixel point in potential target area is belong to a metal target.

Assuming $x$ denotes a pixel to be detected in potential target region, we define the target detection problem as the following two hypotheses:

$H_{0}: x$ belongs to land clutter;

$H_{1}: x$ belongs to target.

According to Bayesian decision theory, GLRT detector is defined as:

$$
\lambda(x)=\frac{p\left(x, \hat{\theta}_{1} \mid H_{1}\right)}{p\left(x, \hat{\theta}_{2} \mid H_{0}\right)} \underset{H_{0}}{\stackrel{H_{1}}{\geq}} \lambda_{T}
$$

where $\hat{\theta}_{1}, \hat{\theta}_{2}$ donate model parameters of Lognormal model and $\mathrm{K}$ distribution respectively, $\lambda_{T}$ is the detection threshold. The optimal threshold can be obtained by averaging repeated detection results. If $\lambda(x) \geq \lambda_{T}$, the current detection obeys $H_{1}$ judgment, otherwise obeys $H_{0}$. We detect potential target region pixel by pixel and output of the result when the detection is completed.

\section{Experimental results and analysis}

In the experiments, the region-based GLRT method is applied to practical target detection in SAR image .And the proposed method is compared with VI-CFAR detection method based on $\mathrm{K}$ distribution and Lognormal distribution

\subsection{Experimental data}

The SAR data used in this experiment comes from the MSTAR program in American. The airborne SAR data is collected with HH-polarization, working at X-band. And the range resolution is $0.3 \mathrm{~m} \times 0.3 \mathrm{~m}$, the cross resolution is $0.2 \mathrm{~m} \times 0.2 \mathrm{~m}$, the image size is 1400 pixels $\times 1400$ pixels. As is shown in Figure 4 (a), the image includes 12 targets to be detected, which are 8 T72 tanks, 4 ZSU23-4 self-propelled guns. The image background is land clutter made of grass and bushes, it is a kind of weak clutter. We get the land clutter region and potential target region after moment-preserving law segmentation and nearest neighbor method process. Then we establish $\mathrm{K}$ distribution model for land clutter region and Lognormal distribution model for potential target region, as shown in Figure 3(a) and 3(b) respectively. From Figure 3, it is easy to learn that the model probability distribution function curve (obtained from the PDF) is very close to the actual amplitude histogram, which illustrates the accuracy of the modeling

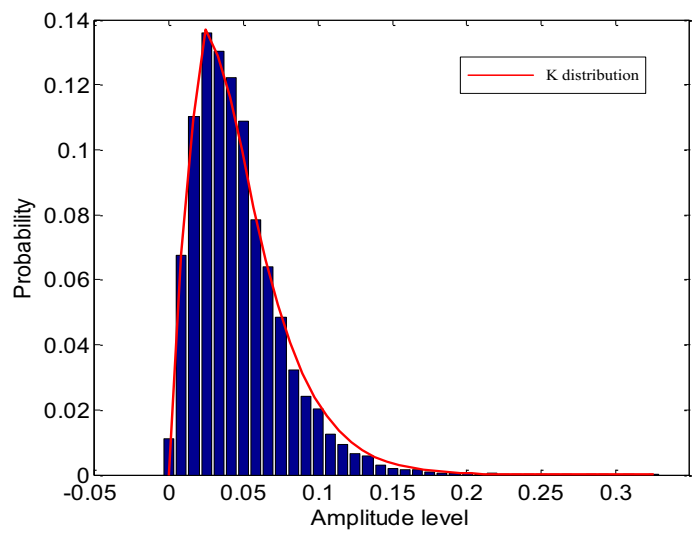

(a) Land clutter region data histogram

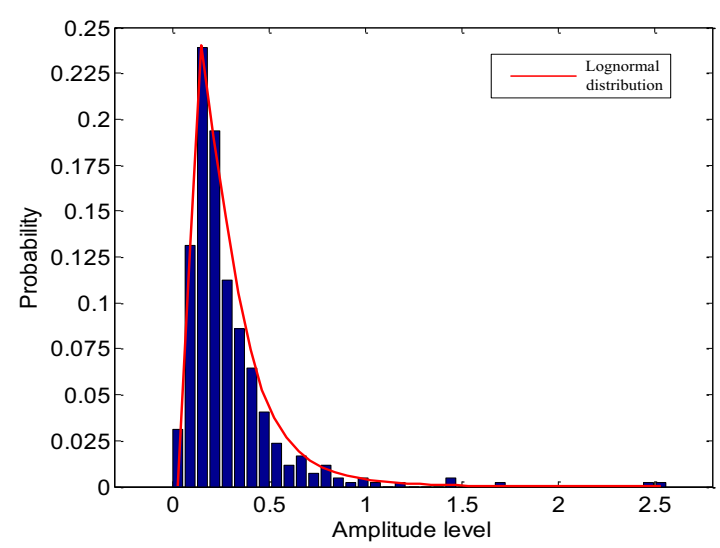

(b) Potential target region data histogram

Fig.3 Data modeling for the region of land clutter and the potential region of metallic target

\subsection{Experimental performance evaluation}

Robertson et al [11] define the quality factor of a detection algorithm as:

$$
F O M=\frac{N_{d t}}{N_{r t}+N_{f a}} \times 100 \%
$$

In formula (7), $N_{d t}$ is the number of true targets; $N_{r t}$ is the number of total targets presented and $N_{f a}$ is the number of false alarms. The closer the Quality factor get to 1 , the better the detection performance is. Also we hope to retain as much of the target information as we can when the target is correctly detected. 


\subsection{Experimental results}

Before the experiment, the target region is identified by artificial vision according to priori knowledge. Then in the follow-up experiments, pixels detected in this region can be considered to be correct, the others are considered to be false alarms pixels. The detection results of proposed method and CFAR method are shown in Figure 4. In the figure, the blocks represent the target vehicles detected correctly. Table 1 shows the detection performance of three methods, in which FOM denotes quality factor of a detection algorithm.

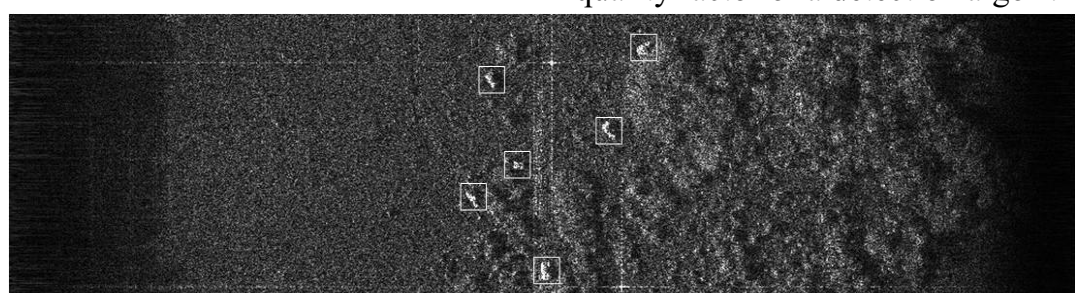

(a) Original SAR image

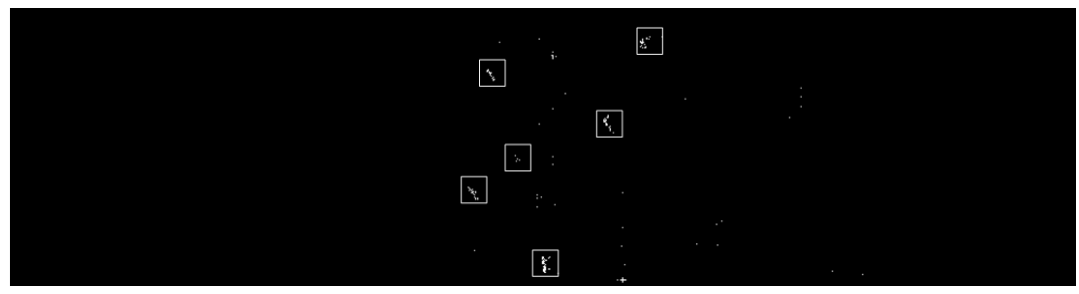

(b) Detection result by proposed method $(\lambda=500)$

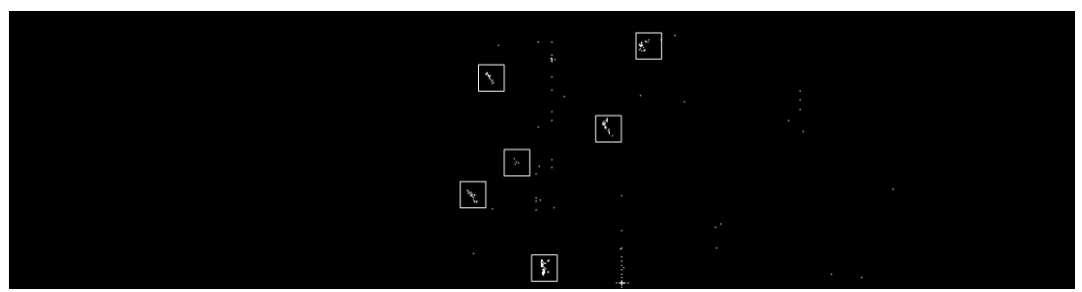

(c) Detection result by VI-CFAR method based on K distribution $\left(P_{f a}=0.00017\right)$

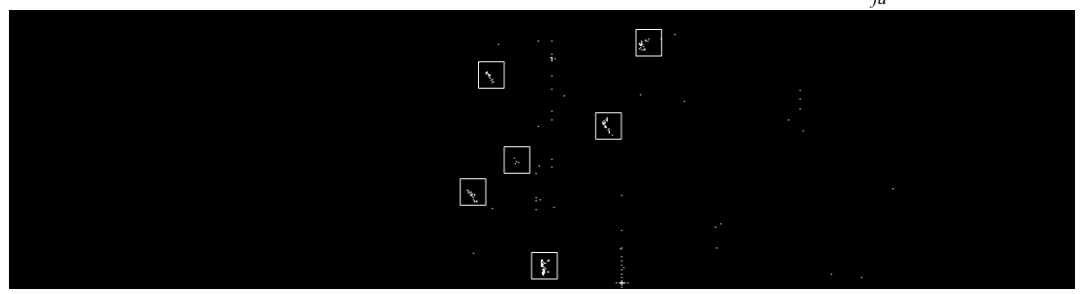

(d) Detection result by VI-CFAR method based on Lognormal distribution $\left(P_{f a}=0.0092\right)$

Fig.4 The detection results based on real SAR data

Table.1 The detection results of the three methods

\begin{tabular}{cccc}
\hline Algorithm & GLRT & $\begin{array}{c}\text { VI-CFAR } \\
\text { (K) }\end{array}$ & $\begin{array}{c}\text { VI-CFAR } \\
\text { (Lognormal) }\end{array}$ \\
\hline Real target & 6 & 6 & 6 \\
Real target & 523 & 523 & 523 \\
pixels & & & \\
$\begin{array}{c}\text { False alarm } \\
\text { pixels }\end{array}$ & 165 & 216 & 223 \\
$\begin{array}{c}\text { Missed target } \\
\text { Running time/s }\end{array}$ & 0 & 0 & 0 \\
\hline
\end{tabular}

The results show that VI-CFAR detection methods based on $\mathrm{K}$ distribution and Lognormal have more false alarms and lower quality factor (between $50 \%$ and $55 \%$ ), which means they can not meet the practical need. The method we proposed have less number of false alarms, also the quality factor is greatly improved (up to
$85.71 \%$ ). This is because GLRT detection algorithm is an optimal Bayesian decision, which uses both of background and target statistics. In addition, VI-CFAR target detection methods based on $\mathrm{K}$ distribution and Lognormal distribution take $302.4 \mathrm{~s}$ and $100.7 \mathrm{~s}$ respectively, while the proposed method only takes 58.3s.In conclusion, the proposed method gets much better accuracy and speed compared to VI-CFAR methods based on the Lognormal distribution $\mathrm{K}$ distribution. The efficiency of target detection is effectively improved.

\section{Conclusion}

The proposed GLRT region-based target detection method is a combination of the conventional image segmentation method and SAR image target detection method. The method firstly roughly segmented to the clutter region and potential target region by using the 
nearest neighbor method and moment retaining method. And then accurate statistical models are established for two regions respectively. Finally the target potential area is detected pixel by pixel by using GLRT target detection method. The experimental results shows that, compared to the VI-CFAR method, the proposed method is improved in terms of detection accuracy and speed, which demonstrates the effectiveness of this method.

\section{References}

1. Oliver C J, Quegan S. Understanding synthetic aperture radar images [M]. Norwood, London Artech House, 1998.

2. Novak L M, Owirka G J, William S, et al. The automatic target-recognition system in SAIP [J]. The Lincoln Laboratory Journal, 1997, 10(2): 187-202.

3. Smith M. E, Varshney P. K. VI-CFAR: A Novel CFAR Algorithm Based on Data Variability. IEEE National Radar Conference, 1997, 263-268

4. Budillon A, Evangelista A, Schirinzi G. GLRT Detection of Moving Targets via Multibaseline Along-Track Interferometric SAR Systems, Geoscience and Remote Sensing Letters, IEEE ,.9(3), May 2012: 348-352

5. Li J, Zelnio E G. Target detection with synthetic aperture radar [J]. IEEE Trans. on AES, 1997, 32(2): 613-627.

6. Perlovsky L I, Schoendorf B J, et al. Model-based neural network for target detection in SAR images [J]. IEEE Transactions on Image Processing, 1997, 6(1): 203-216.

7. Mantero P, Moser G, Serpico S B. Partially supervised classification of remote sensing images using SVM-based probability density estimation [J]. IEEE Trans. on GRS, 2005, 43(3): 559-570.

8. Xie K, Zhou X, Yang P. Assessment of statistical models for clutter and target in SAR images[C]// 29th Chinese Control Conference. Beijing, China: China Academic Journal Electronic Publishing House, 2010: 2997-3002.

9. Roberts W J J, Furui S. Maximum likelihood estimation of K-distribution parameters via the expectation-maximization algorithm [J]. IEEE Transactions on Signal Processing, 2000, 48(12): 3303-3306.

10. Oliver C J. Quegan S. Understanding Synthetic Aperture Radar Images [M]. London: Artech house. 1998

11. Robertson N, Bird P, Brownsword C. Ship Surveillace Using Radarsat ScanSAR Images[C]// Ship Detection in Coastal Waters Workshop 2000. NS, Canada: [s. n.], 2000: 41-45. 